\title{
Global Soil and \\ Sediment Transfers in the Anthropocene (GloSS)
}

\author{
Thomas Hoffmann 1 , D. Penny ${ }^{2}$, V. Vanacker ${ }^{3}$, G. Stinchcomb ${ }^{4}$ and Lu Xixi ${ }^{5}$
}

\author{
Bonn, Germany, 19-21 August 2015
}

The aim of the open kickoff meeting of the PAGES working group GloSS was to set the boundary conditions for GloSS to meet its scientific goals within the next three years. The workshop focused on the development of a list of proxies and indices of human impacts on soil and sediment transfers that will support the compilation of a global soil and sediment database.

A total of 30 participants from different disciplines (geomorphology, geology, soil science, ecology, (paleo)limnology, hydrology, and geoarcheology) and four continents contributed to the workshop.

The first one and a half days were dedicated to continental reviews of proxies of human impacts on soils and sediment transfers. The regional reviews were complemented by a keynote, which provided a global view on sediment production and export, and a videoconference contribution on the experiences of the PAGES $2 k$-team building the PAGES $2 \mathrm{k}$ database.

During the second and third days, the workshop participants (i) developed a concept to organize proxies of human impact on soil and sediment dynamics for hillslopes, floodplains, lakes, and deltas covering different timescales during the Holocene, (ii) discussed the structure, requirements, and potential stakeholders of a GloSS database, and (iii) developed a project road map for the next three years.

The participants of the breakout discussion group on human impact proxies agreed that the first version of the dataset should only in clude proxies and indices of soil erosion and of sediment transport and deposition. Other proxies such as pollen or diatoms, which are not directly related to sediment transport, will not be included. While quantitative volumetric and mass balance proxies are generally favored compared to length-per-time proxies (such as sedimentation or erosion rates given in $\mathrm{mm}$ per year), it was nevertheless noted that the latter are invaluable for reconstructing sediment budgets and that for many study sites complete quantitative inventories are not available. Despite their limitations, length-based rates data will also be collected, but will be interpreted with care due to timescale biases that arise from comparing datasets that average over different timescales. Regarding the considered timescale, the Holocene was considered to be the adequate time frame for the GloSS datasets, as it allows the study of anthropogenic disturbances as well as natural baseline conditions.

The discussion on the GloSS database content and structure resulted in the following statement: The database should focus on soil

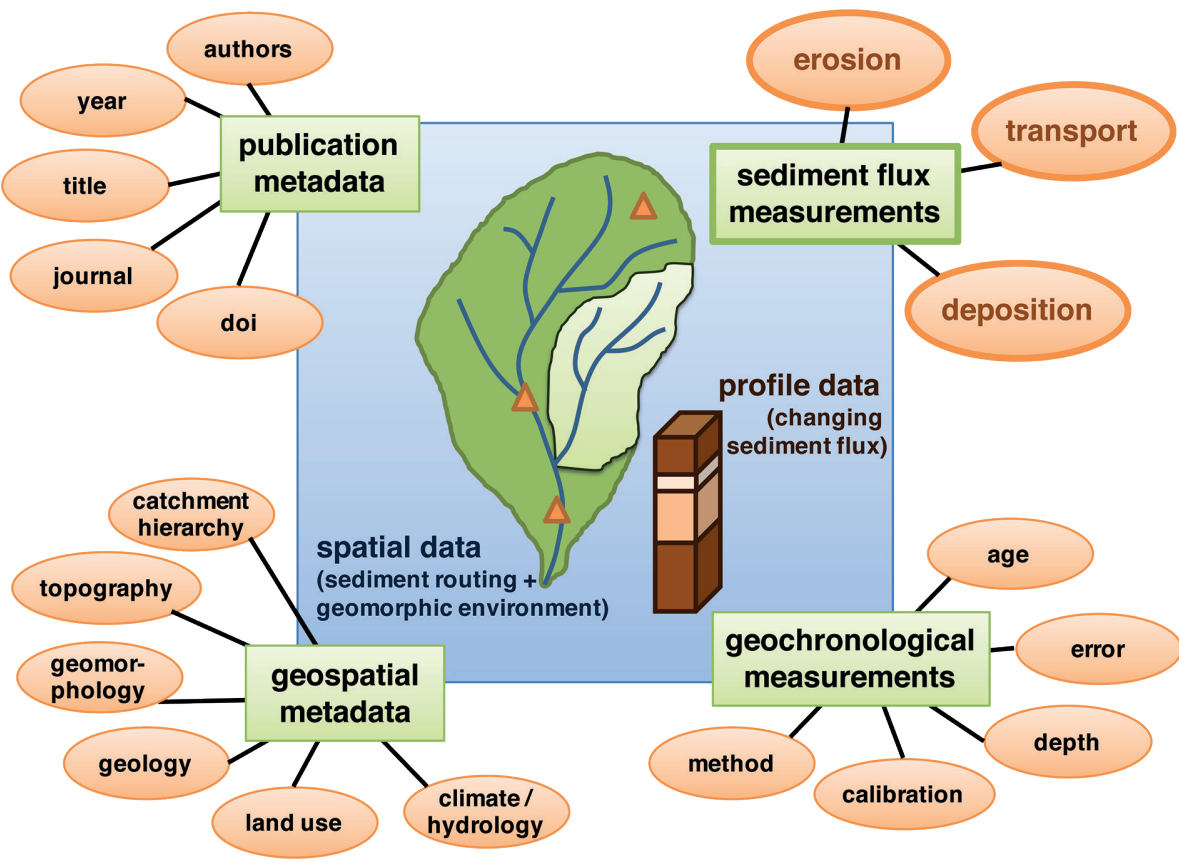

Figure 1: Planned structure of the global GloSS database focusing on human impact on soil erosion, sediment transport and deposition during the Holocene. erosion and sediment transport and deposition of hillslopes, floodplains, and channels, lakes, and deltas (Fig. 1). At this point, there is no need to populate the database with other available paleo-environmental information. Instead, the database should be linked to other existing databases to avoid redundancy where possible.

The participants agreed that human impact on the above-mentioned environments is best described by the changes in erosion, transport, and deposition. Thus, at minimum, two time slices, before and after human impact for those three variables, are necessary. Due to the major challenges of defining what human impact actually is and when it starts in different regions of the earth, it was argued that the number of time slices should be larger to allow flexible interpretations without constraining what the user can interpret from inferred changes (e.g. climate versus human impacts).

To set the boundary conditions for the first three years of the GloSS working group, a roadmap, seven regional task forces, and a database task force were built.

The regional task forces and their leaders are: Europe and Mediterranean (Gert Verstraeten), South Asia (Rajiv Sinha), SE Asia (Dan Penny), Australia and New Zealand (Bob Wasson, Duncan Cook), East and Central Asia (Hongming He, Lu Xixi), North America (Jane Willenbring, Gary Stinchcomb), South and Central America (Juan Restrepo), and Africa (Klaus Martin Moldenhauer, to be confirmed).

The database task force includes Jane Willenbring, Gary Stinchcomb, Veerle Vanacker, Dan Penny, Lishan Ran, Jean Philippe Jenny, Nick Mackay, Kim Cohen, and Thomas Hoffmann.

If you would like to participate in any of these activities, visit the GloSS webpage at (www. pages-igbp.org/ini/wg/gloss).

\section{AFFILIATIONS}

'Department of Geography, University of Bonn, Germany

${ }^{2}$ School of Geosciences, University of Sydney, Australia ${ }^{3}$ Earth and Life Institute, Université catholique de Louvain, Louvain-la-Neuve, Belgium

${ }^{4}$ Department of Geosciences, Murray State University, Murray, USA

${ }^{5}$ Department of Geography, National University of Singapore, Singapore

\section{CONTACT}

Thomas Hoffmann: thomas.hoffmann@bafg.de 